\title{
Die Entwicklungsstufen von Künstlicher Intelligenz
}

Wir stehen heute an dem Punkt, dass Maschinen nicht nur anhand von Schlüsselbegriffen (Keywords) verstehen, was eine Person möchte, sondern sie auch eigenständig versuchen in Erfahrung zu bringen, was das Ziel eines Menschen ist. Der nächste Schritt ist, daraus auch eigenständig Aktionen abzuleiten. Das gelingt allerdings heute noch nicht ohne Reibung. Bei all der Entwicklung von Künstlicher Intelligenz $(K I)$ ist es erstrebenswert, weiterhin den Menschen final über abzuleitende Aktionen entscheiden zu lassen und nicht die Maschine.

\section{Künstliche Intelligenz basiert im Prinzip auf Wahrscheinlichkeitsrech-} nung und gibt entsprechende Wahrscheinlichkeiten aufgrund erkannter Muster und Kategorien an den Entwickler aus. Je nachdem, wie hoch der erreichte Prozentsatz der Trefferquote in der Wahrscheinlichkeit ist, entscheidet der Entwickler heute, ob er den erkannten Intent durch- oder nicht durchlässt. In Machine und Deep Learning liegen natürlich jede Menge Chancen, aus ungeheuren Datenmengen, die das menschliche Hirn nicht in der Lage wäre zu sortieren, geschweige denn nach Mustern zu durchsuchen, Regelmäßigkeiten zu entdecken und anhand dessen in wahrscheinliche Kategorien einzuordnen.

Wenig verwunderlich ist es daher, dass vor allem im Finanz- und Medizinbereich derzeit solche Systeme zunehmend Anwendung finden. Die Frage, die am Ende immer mit Ja beantwortet werden sollte, ist doch, ob die Technologie uns das Leben erleichtert und uns hilft oder eher das Gegenteil bewirkt. Richtig eingesetzt kann sie uns helfen, falsch eingesetzt ist sie gefährlich wie ein jedes Küchenmesser auch. 